induced contrasts was varied between trials and the participant was required to judge the angle on each trial (Figure 1). Hence, the task mandated a fine-scaled decision which required detail to be extracted detail at such a fine scale that it may only be present in V1 due to its small receptive fields - and might therefore have induced correlated V1 activity.

A hypothesis put forth by Crick and Koch in their 1995 paper was that, while activity in V1 may be necessary for conscious perception, it may not be sufficient. According to Crick and Koch, sensory regions need to be connected to prefrontal cortex to enable conscious perception. Whether involvement of frontal regions is necessary remains controversial (Eriksson et al., 2008), but a role of prefrontal cortex is indicated by a large number of neuroimaging studies showing a correlation between activity in frontal regions and conscious perception (Naghavi and Nyberg, 2005; Rees et al., 2002). In the Maertens et al. study, a limited part of the brain was sampled to improve spatial resolution. While this enabled the retinotopic mapping of the IC and thus constituted a great strength of study, this level of detail comes with a cost as nothing could be concluded regarding frontal involvement in the creation of IC. Future work, building on the methods used in the Maertens et al. study along with whole-brain sampling could shed light on the involvement of higher-order cortical regions and necessary vs. sufficient conditions for conscious perception.

In sum, the Maertens et al. study is an excellent demonstration of the constructive nature of perception (Helmholtz, 1910). Moreover, it shows that this constructive process is not confined to higher levels of the visual hierarchy, but can take place even at the lowest level of cortical processing.

\section{REFERENCES}

Crick, F., and Koch, C. (1995). Are we aware of neural activity in primary visual cortex? Nature 375 , 121-123.

Eriksson, J., Larsson, A., and Nyberg, L. (2008). Item-specific training reduces prefrontal cortical involvement in perceptual awareness. J. Cogn. Neurosci. 20, 1777-1787.
Hochstein, S., and Ahissar, M. (2002). View from the top: hierarchies and reverse hierarchies in the visual system. Neuron 36, 791-804.

Maertens, M., Pollmann, S., Hanke, M., Mildner, T., and Möller, H. E. (2008). Retinotopic activation in response to subjective contours in primary visual cortex. Front. Hum. Neurosci. 2. doi: 10.3389/ neuro.09.002.2008. [Epub ahead of print].

Naghavi, H. R., and Nyberg, L. (2005). Common fronto-parietal activity in attention, memory, and consciousness: shared demands on integration? Conscious. Cogn. 14, 390-425.

Rees, G. (2007). Neural correlates of the contents of visual awareness in humans. Philos. Trans. R. Soc. Lond., B, Biol. Sci. 362, 877-886.

Rees, G., Kreiman, G., and Koch, C. (2002). Neural correlates of consciousness in humans. Nat. Rev. Neurosci. 3, 261-270.

Tong, F. (2003). Primary visual cortex and visual awareness. Nat. Rev. Neurosci. 4, 219-229.

von Helmholtz, H. (1910). Handbuch der physiologischen optik, Vol. 3, $3^{\text {rd }}$ Edn. Leipzig, Leopold Voss.

Received: 28 April 2009; published: 15 September 2009. Citation: Front. Neurosci. (2009) 3, 2: 159-160. doi 10.3389/neuro.01.018.2009

Copyright (c) 2009 Eriksson and Nyberg. This is an openaccess publication subject to an exclusive license agreement between the authors and the Frontiers Research Foundation, which permits unrestricted use, distribution, and reproduction in any medium, provided the original authors and source are credited.

\title{
Calcium waves in astrocyte networks: theory and experiments
}

\section{Michele Giugliano ${ }^{1,2 *}$}

1 Department of Biomedical Sciences, University of Antwerp, Wirrijk, Belgium

2 Laboratory of Neural Microcircuitry, Brain Mind Institute, EPFL, Lausanne, Switzerland

*Correspondence: michele.giugliano@ua.ac.be

\section{A commentary on}

Diffusion modeling of ATP signaling suggests a partially regenerative mechanism underlies astrocyte intercellular calcium waves

by Christopher L. MacDonald, Diana Yu, Marius Buibas and Gabriel A. Silva

In the central nervous system (CNS), astrocytes participate in supportive functions, such as metabolism, ion homeostasis, neurotransmitter recycling and tissue integrityrestoration. However, astrocytes are also deeply involved in a variety of complex phe- nomena, including CNS physiology, information processing, and synaptic plasticity (Fellin, 2009). These make the investigation of astrocytes (dis)functions as challenging as of neurons and synapses.

Interestingly, astrocytes are excitable cells like neurons. They base their communication on spontaneous or evoked cytosolic $\mathrm{Ca}^{2+}$ variations, instead of membrane electrical transients. Their remarkable morphology supports intercellular signaling as they form interconnected networks of cells coupled by gap-junctions, where each unit occupies a virtually non-overlapping domain of the inter-neuronal space. Surprisingly, astrocytes communicate also to neurons and synapses. In fact, they extend membrane processes to simultaneously contact hundreds of neuronal dendrites, thousands of synapses and even blood vessels. Indeed, astrocytes control the vasculature tone and they are likely to sense neuronal energydemand and gate its consumption. Their physiology is thus bidirectionally linked to neuronal and synaptic activity, as they are capable of selectively respond to it on a millisecond time scale, by releasing specific neuroactive molecules (Ni et al., 2007). Notable are the discoveries of the interaction with synaptic physiology and plasticity that led 
to revisiting information transfer between neurons, with the proposed concept of a "tripartite synapse" (Perea and Araque, 2002).

In this issue, MacDonald et al. (2008) focus on the astrocyte-to-astrocyte communication, by exploring the long-range propagation mechanisms of intracellular calcium waves (ICW). These represent a form of intercellular signaling in astrocyte networks, where ATP release is initiated by the intracellular $\mathrm{Ca}^{2+}$ elevation, and whose diffusion acts as cell-to-cell transmitter. Authors employ in vitro calcium imaging in astrocytes cultures and a simple phenomenological mathematical model. Through such a combined approach, they examine two apparently conflicting scenarios, as debated in the literature: during ICW, (1) ATP is secreted by one cell (i.e., a point-source) and diffuses activating nearby astrocytes, or (2) ATP-induced cell activation is actively regenerated by downstream astrocytes, which in turn secrete ATP, similarly to propagation of an action-potential through a myelinated axon.

Only through a combination of (1) and (2), resulting in both regenerative and diffusive mechanisms, the model is able to match quantitatively the in vitro ICW. This result reconciles elegantly the previous debate between single-point source models and fully regenerative signaling models, and it is particularly significant due to the extremely simple nature of the model. Even though several biophysical details underlying ATP diffusion and single-cell "excitability" were neglected, this model allows for systematic interpretation and analysis of the relationship between single-cell and network emerging properties, which is hard to obtain in accurate biophysical descriptions.

It is intriguing to outline the similarity between the astrocyte model introduced here, and the spiking network models of the integrate-and-fire family. The last are reduced models of neuronal excitability, shown to capture quantitatively the experimental responses from cells in large networks (Jolivet et al., 2008). Similarly, MacDonald et al. (2008) identifies a minimal description to predict network-level ICW propagation. These considerations therefore suggest that the two modeling approaches may be unified, incorporating spiking neurons, chemical synapses as well as astrocytes, ICW and ATP-diffusion. The results of such attempts might be valuable to identify global activity regimes, to be explored in greater details through large-scale simulations (Markram, 2006) that incorporate anatomically precise details on neurons, astrocytes and the intercellular environment (Helmstaedter et al., 2009).

\section{REFERENCES}

Fellin, T. (2009). Communication between neurons and astrocytes: relevance to the modulation of synaptic and network activity. J. Neurochem. 108, 533-544.

Helmstaedter, M., Briggman, K. L., and Denk, W. (2009). $3 \mathrm{D}$ structural imaging of the brain with photons and electrons. Curr. Opin. Neurobiol. 18, 633-641.

Jolivet, R., Schürmann, F., Berger, T. K., Naud, R., Gerstner, W., and Roth, A. (2008). The quantitative single-neuron modeling competition. Biol. Cybern. 99, 417-426.

MacDonald, C. L., Yu, D., Buibas, M., and Silva, G. A. (2008).Diffusion modeling of ATP signaling suggests a partially regenerative mechanism underlies astrocyte intercellular calcium waves. Front. Neuroeng. doi: 10.3389/neuro.16.001.2008.

Markram, H. (2006). The blue brain project. Nat. Rev. Neurosci. 7, 153-160.

Ni, Y., Malarkey, E. B., and Parpura, V. (2007). Vesicular release of glutamate mediates bidirectional signaling between astrocytes and neurons. J. Neurochem. 103, 1273-1284.

Perea, G., and Araque, A. (2002). Communication between astrocytes and neurons: a complex language. J. Physiol Paris 96, 199-207.

Received: 04 May 2009; published: 15 September 2009. Citation: Front. Neurosci. (2009) 3, 2: 160-161. doi 10.3389/neuro.01.019.2009

Copyright (c) 2009 Giugliano. This is an open-access publication subject to an exclusive license agreement between the authors and the Frontiers Research Foundation, which permits unrestricted use, distribution, and reproduction in any medium, provided the original authors and source are credited.

\section{Genes and cognitive aging}

\section{William Jagust*}

Helen Wills Neuroscience Institute, University of California, Berkeley, Berkeley, CA, USA

*Correspondence: jagust@berkeley.edu

\section{A commentary on}

Genetic contributions to age-related decline in executive function: a 10-year longitudinal study of COMT and BDNF polymorphisms

by Kirk I. Erickson, Jennifer S. Kim, Barbara L. Suever, Michelle W. Voss, B. Magnus Francis and Arthur F. Kramer

The search for genetic factors related to late life cognitive change initially focused on cognitive diseases, particularly Alzheimer's disease (AD) and now increasingly employs the method of genome-wide association studies. This approach has repeatedly confirmed the major importance of the Apolipoprotein E polymorphism as a risk factor for $\mathrm{AD}$, but no consensus has emerged concerning other genes. The reasons for this situation likely include factors such as the large number of potential candidate genes with small effects, interactions between genes, and the difficulty of establishing a reli- able and valid phenotypic marker of both disease and normality.

The field of cognitive aging is also grappling with the genetic determinants of cognitive change, using different approaches in the face of a unique set of challenges. Because cognitive aging is probably even more heterogeneous than a cognitive disorder like $\mathrm{AD}$, definition of the precise phenotype is problematic. Factors that can define this phenotype include the exact cognitive process and a host of demographic factors. In addition, the small effect sizes and poten- 